\title{
Eficacia de un programa de entrenamiento a padres para la mejora del bienestar psicológico y la satisfacción familiar: Educar con una sonrisa ("Parenting with a Smile")
}

\author{
Carmen Pérez ${ }^{1}$ y Marina Iniesta ${ }^{2}$ \\ ${ }^{1} \mathrm{CIO}$ centro Psicología, Murcia (España); ${ }^{2}$ Universidad Católica de Murcia (España)
}

\begin{abstract}
El objetivo del presente estudio consistió en diseñar, aplicar y analizar la eficacia del programa de entrenamiento para padres Educar con una sonrisa como modelo estructurado de intervención psicoeducativa destinado a la disminución de la sintomatología ansiosa, depresiva y el estrés a través del aumento de la satisfacción familiar y la instrucción en habilidades de crianza y cambio de actitud en padres de hijos de entre 0 y 12 años. Se llevó a cabo un estudio cuasi-experimental con una muestra de 68 padres. Se realizó una evaluación pretest- postest de la sintomatología clínica de los padres relacionada con la ansiedad, la depresión y el estrés a través de la Escala Abreviada de Depresión, Ansiedad y Estrés (DASS-21), de la satisfacción familiar según la Escala de Satisfacción Familiar por Adjetivos (ESFA) y de las habilidades y dimensiones cognitivas en relación con la crianza mediante el Cuestionario para la Evaluación de Adoptantes, Cuidadores, Tutores y Mediadores (CUIDA). El test de Wilcoxon mostró que las reducciones en las puntuaciones de la sintomatología clínica y los incrementos en la satisfacción familiar y habilidades de crianza, salvo para Altruismo, son significativas.
\end{abstract}

Palabras clave: Programas de entrenamiento, satisfacción familiar, crianza, habilidades parentales.

Effectiveness of a parent training program aiming to improve the psychological wellbeing and the family satisfaction: Parenting with a Smile. The purpose of this study was to design, implement and analyse the effectiveness of the parent training program Parenting with a smile, taking it as a structured role model in a psychoeducational intervention which aims to reduce anxiety, depression and stress symptoms by increasing the family satisfaction, the training in parenting skills and the change in attitude from parents with children aged between 0 and 12 . A quasi-experimental study with a sample of 68 parents was carried out along with a pretest-posttest evaluation of the parents' clinical symptoms in relation to anxiety, depression and stress by using the Abreviated Depression Anxiety Stress Scale [Escala Abreviada de Depresión, Ansiedad y Estrés (DASS-21)], of the family satisfaction following the Family Satisfaction by Adjectives Scale (FSAS) [Escala de Satisfacción Familiar por Adjetivos (ESFA)] and finally of the skills and cognitive dimensions related to parenting through the Questionnaire for the assessment of adopters, carers, tutors and mediators [Cuestionario para la Evaluación de Adoptantes, Cuidadores, Tutores y Mediadores (CUIDA)]. The reductions in clinical symptoms scores and the increase in family satisfaction and parenting skills, except for Altruism, shown in Wilcoxon test are significant.

Keywords: Training programs, family satisfaction, parenting, parenting skills, psychological wellbeing.

Correspondencia: Carmen Pérez-Saussol. CIO Centro Psicología. Plaza Puerta Nueva 3, $\mathrm{n}^{\circ} 1$. Esc. bajo B, C.P. 30008. Murcia (España). E-mail: carmensau@gmail.com 
La familia se ha considerado tradicionalmente como un núcleo generador de bienestar (Manuel, Casanova, Carpio, y Cerezo, 2015). Sin embargo, diversos estudios han demostrado los efectos negativos que puede generar la crianza sobre la salud mental de los padres. La entrada en la paternidad se considera como una fuente importante de estrés (Padilla, Lara, y Álvarez-Dardet, 2010) y posible generadora de sintomatología ansiosa y/o depresiva (Pérez y Santelices, 2016). Tales estados psicopatológicos afectan a la calidad de la crianza y, a su vez, al desarrollo psicosocial y a la autorregulación de las conductas agresivas en los hijos (Raya, Pino, y Herruzo, 2009). Es por ello, que la calidad de las relaciones socio-afectivas entre padres e hijos durante la crianza ha sido relacionada con el ajuste psicológico, el desarrollo y la salud de los hijos (Maggi, Irwin, Siddiqi, y Hertzman, 2010). En esta misma línea de investigación, diversos autores han constatado la relación existente entre la presencia de sintomatología psicopatológica en los padres y el aumento en la probabilidad de padecer problemas psicológicos en los hijos (Bloomfield y Kendall, 2012; Leijdesdorff, van Doesum, Popma, Klaassen, y van Amelsvoort, 2017). Jones, Lebowitz, Marin, y Stark (2015) indicaron que los padres con elevados niveles de ansiedad manifestaban comportamientos que fomentaban la aparición de sintomatología ansiosa en sus hijos. Middeldorp et al. (2016) estudiaron el nivel de severidad de la sintomatología ansiosa y depresiva en los padres como factor relevante en la respuesta psicopatológica de los niños. En consecuencia, la falta de salud mental de los padres constituye un factor de riesgo con implicaciones negativas en la calidad de las relaciones familiares y es determinante, en buena medida, en el desarrollo presente y futuro de los hijos.

El malestar psicológico asociado al proceso de crianza se encuentra asociado a diversos factores detonantes: (1) La crianza supone para los padres una tarea compleja que requiere de nuevas habilidades, recursos y fuentes de apoyo (Rodrigo, Almeida, Spiel, y Koops, 2012). (2) La ausencia de modelos referenciales y el acceso a información no contrastada en situaciones conflictivas durante la crianza puede dificultar el desempeño de unas adecuadas prácticas de crianza, generar mitos y expectativas falsas (Flores-Bravo, Valadez-Sierra, Rosal, y Betancourt-Morejón, 2018). (3) Las emociones, creencias y actitudes disfuncionales negativas suelen aflorar durante la maternidad y éstas pueden afectar negativamente al proceso de crianza (Crandall, Deater-Deckard, y Riley 2015; Otani et al, 2013). (4) La pérdida del control educativo sobre los hijos es algo frecuente durante la crianza (Sánchez, 2017). (5) El mantenimiento de un estado de alarma, preocupación, duda, auto-exigencia e incertidumbre suele ser un factor de malestar en las figuras parentales (López de Arana-Prado y Barandiaran-Arteaga, 2018). (6) La ausencia de dimensiones psicológicas parentales como la apertura, la capacidad para resolver problemas, la empatía, el equilibrio emocional, la sociabilidad, la tolerancia a la frustración y la capacidad para establecer vínculos afectivos han sido asociadas de manera significativa al desgaste psicológico en los cuidadores 
(Pérez-Adame, Fulgencio-Juárez, y González-Zepeda, 2013). (7) La subordinación de las necesidades básicas de los padres a las de los hijos, como causa de malestar parental ante la sensación de límite de libertad y posibilidades de crecimiento profesional, fue recogida en una encuesta realizada por el Centro de investigaciones sociológicas (CIS, 2012).

Figura 1. Modelo explicativo propuesto para el proceso de crianza desadaptativo

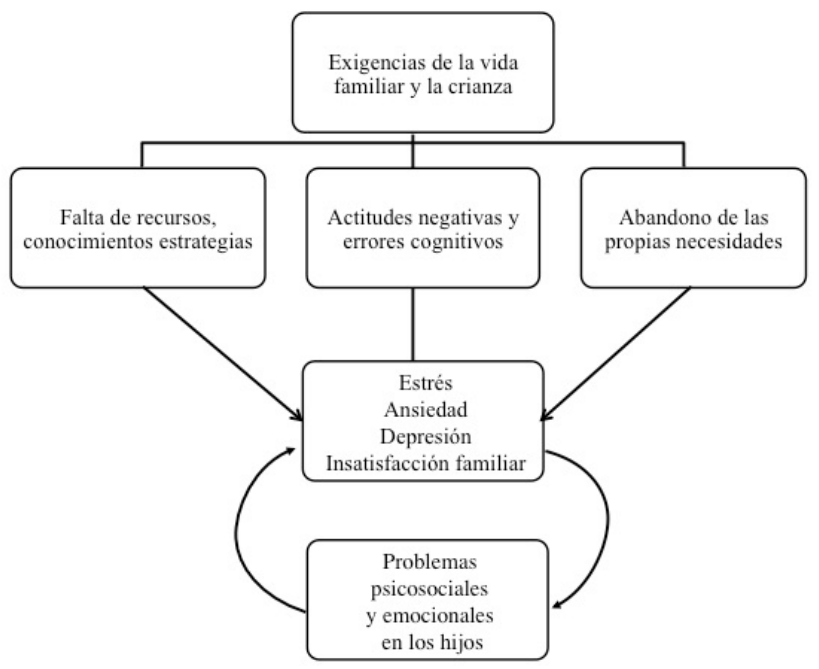

De acuerdo con Armstrong, Birnie-Lefcovitch, y Ungar, (2005) y Pérez y Santelices (2016), las exigencias de la vida familiar, la crianza, los problemas relacionados con la salud, el comportamiento de los hijos, la gestión del tiempo, la economía, etc. llevaría a los padres a experimentar una sensación de falta de recursos, conocimientos y estrategias para hacer frente a las diferentes situaciones problemáticas que presentan. Este tipo de situaciones suelen actuar como desencadenante de conductas desadaptativas en los padres provocando problemas de tipo emocional y comportamental en los hijos y generando a su vez un incremento mayor del malestar parental, ocasionando un proceso cíclico y bidireccional de desajuste tanto para los padres como para los hijos (Furlong et al., 2012; Shlonsky et al., 2016).

Teniendo en cuenta la posibilidad de mejora de la salud psicológica en los padres y un aumento en el ajuste de los hijos durante la crianza, se propone un modelo explicativo sobre la aparición de síntomas psicopatológicos en los padres y su relación con los problemas psicosociales en los niños. La figura 1 refleja cómo las exigencias de la crianza provocan sintomatología clínica asociada a la ansiedad, la depresión y el estrés 
a causa de la falta de recursos, conocimientos y estrategias; a la presencia de actitudes negativas y errores cognitivos; y al abandono de las propias necesidades de los padres. Esta sintomatología presente en las figuras parentales repercute en el desarrollo psicosocial y emocional de los hijos, revirtiendo en la sintomatología de los padres de manera cíclica y convirtiendo la etapa de crianza en un proceso disfuncional como indicó Furlong et al. (2012) y Shlonsky et al. (2016).

Gracias a los estudios realizados por Patterson y colaboradores sobre los programas de entrenamiento a padres (Patterson, 1982; Patterson, Reid, Jones, y Conger, 1975), surgieron varias líneas de investigación dirigidas a validar los programas de entrenamiento como una intervención clínica alternativa a la psicoterapia tradicional, encontrando tras sus resultados ventajas de coste y de eficacia (McMahon, 1991). A parte de constatar estos beneficios originados principalmente por su implementación grupal, Godoy, Ruiz-Castilla, Fresneda-López, y Puertas-González (2019) incorporaron como ventajas de los programas de entrenamiento sobre las terapias tradicionales (1) el empoderamiento que reciben las figuras parentales al ser dotados de la capacidad de intervención de problemas de comportamiento y (2) la posibilidad de trabajar en un ambiente natural para el niño. Tosh, Arnott, y Scarinci, (2017) equipararon el nivel de eficacia de los programas de entrenamiento a las terapias tradicionales, siempre que estos proporcionasen una dosis y una intensidad similar a las terapias tradicionales. Tras haber evidenciado que los programas de entrenamiento a padres mejoran las prácticas parentales y el ajuste emocional y comportamental de los hijos (Barlow, Smailagic, Ferriter, Bennett, y Jones, 2016). Lozano-Rodríguez y Valero-Aguayo (2017) realizaron una revisión sistemática sobre la eficacia de los programas de entrenamiento a padres en relación con: 1) La metodología utilizada, en cuyos resultados señalaron una utilización generalizada de escalas y cuestionarios autoaplicables por encima de observaciones directas y registro de videos. 2) El tipo de problemática, siendo los más utilizados los programas cuyo objetivo consiste en mejorar el comportamiento de los hijos, seguidos de los programas centrados en la instrucción en habilidades parentales para la adquisición de competencias relacionadas con el aumento de responsabilidades y la mejora en la relación con los hijos, y en tercer lugar, aquellos programas cuyo objetivo consistía en aumentar las competencias parentales de padres de hijos con problemas específicos. 3) El tamaño del efecto, medido con la $d$ de Cohen, utilizada para detectar las mejoras más efectivas y cuyos resultados resaltaron el comportamiento, las conductas agresivas de los hijos y el funcionamiento familiar. 4) Los tratamientos eficaces, a pesar de la dificultad para clasificarlos según el nivel de eficacia científica presentada (Chambless y Hollon, 1998); Lozano-Rodríguez y Valero-Aguayo (2017) determinaron que los programas de entrenamiento de mayor incidencia sobre la conducta de los hijos fueron: el programa Parents Plus Children's Programme (Mendoza, Pedroza, y Martínez, 2014), el programa 1,2,3 Magic Parenting Program (Porzing-Ddrummong., 
Stevenson, y Stevenson., 2014, 2015), Incredible Years (Eamesa et al., 2010) y el programa Parent Management Training y The Oregon Model (Kjøbli y Ogden, 2009; Kjøbli, Hukkelberg, y Ogden, 2013). El programa Home Start (Hermanns, Assecher, Zijlstra, Hoffenaar, y Dekovic., 2013, Van Aar., Asscher, Zijlstra, Dekovic, y Hoffenaar, 2015) también fue destacado en cuanto a su eficacia para la mejora en la vida familiar y en la crianza de los hijos. A su vez, Home Start (Hermanns et al., 2013, Van Aar et al., 2015) e Incredible Years (Eamesa et al., 2010) fueron considerados los más relevantes para la organización metodológica y la mejora en el comportamiento de los hijos.

A pesar de que la literatura científica ha sido capaz de demostrar la eficacia de los programas de entrenamiento para la mejora y prevención de problemas de comportamiento (González, Castilla, López, y Godoy, 2019), la línea de investigación dirigida a la incidencia de los programas de entrenamiento a padres sobre el aumento de la salud psicológica de las figuras parentales no ha sido tan extensa como para presentar datos concluyentes. Bennett, Barlow, Huband, Smailagic, y Roloff (2013) realizaron un meta-análisis en el que detectaron que asistir a este tipo de programas de entrenamiento producía un efecto positivo modesto sobre la sintomatología clínica de los padres relacionada con la ansiedad, la depresión y el estrés. Igualmente, se observaron mejoras psicológicas así como, un aumento en las habilidades y dimensiones cognitivas relacionadas con el descenso de ira y culpa en los padres. Este tipo de estudios muestra contradicciones ya que existen investigaciones en las que no se ha hallado ningún efecto significativo de los programas de entrenamiento a padres sobre los síntomas psicopatológicos en las figuras parentales (Hiscock et al., 2008; Martin y Sanders, 2003; Matsumoto, Sofronoff, y Sanders, 2010). Esta ausencia de evidencia significativa en algunos estudios se atribuiría a que los programas de entrenamiento hasta la fecha se dirigían principalmente a enseñar a los padres técnicas y estrategias para el manejo de la conducta del niño, como se aprecia en el programa Triple P-Positive Parenting Program (Sanders, Turner, y Markie-Dadds, 2002). Otros programas como Incredible Years Parent Training (IYPT; Webster-Stratton, 2001) a pesar de combinar estrategias cognitivo-conductuales para identificar y cambiar patrones distorsionados de creencias y pensamientos, no centran su intervención, principalmente, en el aumento de la salud psicológica de los padres, ni en su satisfacción familiar.

Ante la falta de este tipo de investigaciones en relación a la salud psicológica de los padres se propone el programa de entrenamiento a padres Educar con una sonrisa elaborado tras la publicación del libro Educar con una sonrisa (Perez-Saussol, 2015). Este programa se construye con el objetivo principal de aumentar la salud psicológica de los padres y su satisfacción familiar para así conseguir instruir de manera eficaz a las figuras parentales en habilidades y competencias cognitivas en relación a la crianza. El presente artículo expone los resultados de la evaluación realizada sobre la eficacia de dicho programa para la mejora de la sintomatología clínica relacionada con la ansiedad, 
la depresión y el estrés, la satisfacción familiar, así como, el aumento de diversas habilidades y dimensiones cognitivas relacionadas con el proceso de crianza.

\section{MÉTODO}

\section{Participantes}

Se establecieron como criterios de inclusión ser mayor de 18 años de edad y tener al menos un hijo de edad comprendida entre los 0 y los 12 años, y como criterios de exclusión se determinó que los padres no debían presentar ninguna condición que pudiera limitar el entendimiento del proceso de intervención y evaluación, ni tener hijos con enfermedades crónicas, retraso grave en el desarrollo o trastornos. Para formar parte de la muestra se impuso como requisito que los padres debían haber acudido a todas las sesiones de entrenamiento, haber rellenado y entregado correctamente las evaluaciones realizadas y no haber tenido un retraso en la asistencia a ninguna de las sesiones de más de 10 minutos.

Tabla 1. Estado civil, sexo y edad de los participantes

\begin{tabular}{lccccccc}
\hline Características de los padres & \multicolumn{2}{c}{$\begin{array}{c}\text { MUJERES } \\
(\mathrm{n}=55,80.9 \%)\end{array}$} & $\begin{array}{c}\text { HOMBRES } \\
(\mathrm{n}=13,19.1 \%)\end{array}$ & \multicolumn{2}{c}{$\begin{array}{c}\text { TOTAL } \\
(\mathrm{N}=68,100 \%)\end{array}$} \\
\hline & Media & $D T$ & Media & $D T$ & Media & $D T$ \\
\hline Edad & 39.2 & 4.11 & 42.62 & 5.12 & 39.88 & 4.30 \\
\hline & $n$ & $\%$ & $n$ & $\%$ & $n$ & $\%$ \\
\hline \multirow{2}{*}{ E. Civil } & Casado o en Pareja & 51 & $92.73 \%$ & 13 & $100 \%$ & 64 & $94.12 \%$ \\
\cline { 2 - 9 } & Separado, divorciado o viudo & 4 & $7.27 \%$ & 0 & .00 & 4 & $5.88 \%$ \\
\hline
\end{tabular}

Tabla 2. Características relacionadas con los hijos

\begin{tabular}{|c|c|c|c|c|c|c|c|}
\hline & & Media & $D T$ & Media & $D T$ & Media & $D T$ \\
\hline Número de hijos & & 1.96 & 0.74 & 2.00 & 0.40 & 1.97 & 0.69 \\
\hline \multirow[t]{2}{*}{ Edad de los hijos } & & 7.15 & 4.89 & 6.92 & 4.18 & 7.10 & 4.74 \\
\hline & & $n$ & $\%$ & $n$ & $\%$ & $n$ & $\%$ \\
\hline \multirow{4}{*}{ Número de hijos } & 1 hijo & 15 & $27.27 \%$ & 1 & $7.69 \%$ & 16 & $23.53 \%$ \\
\hline & 2 hijos & 28 & $50.91 \%$ & 11 & $84.62 \%$ & 39 & $57.35 \%$ \\
\hline & 3 hijos & 11 & $20.00 \%$ & 1 & $7.69 \%$ & 12 & $17.65 \%$ \\
\hline & 4 hijos & 1 & $1.82 \%$ & 0 & $0.00 \%$ & 1 & $1.47 \%$ \\
\hline \multirow{4}{*}{ Edad de los hijos } & $0-4$ & 34 & $31.48 \%$ & 9 & $34.62 \%$ & 44 & $32.09 \%$ \\
\hline & $5-8$ & 39 & $36.11 \%$ & 7 & $26.92 \%$ & 45 & $34.33 \%$ \\
\hline & $9-12$ & 24 & $22.22 \%$ & 7 & $26.92 \%$ & 31 & $23.13 \%$ \\
\hline & $>12$ & 11 & $10.19 \%$ & 3 & $11.54 \%$ & 14 & $10.45 \%$ \\
\hline \multirow{2}{*}{ Sexo de los hijos } & Varones & 62 & $59.62 \%$ & 20 & $76.92 \%$ & 85 & $61.19 \%$ \\
\hline & Mujeres & 46 & $44.23 \%$ & 6 & $23.08 \%$ & 49 & $38.81 \%$ \\
\hline
\end{tabular}

El programa Educar con una sonrisa fue implementado en dos colegios, una empresa, una guardería y un centro de psicología de la Región de Murcia. De los 98 padres que comenzaron el programa de entrenamiento, 68 configuraron la muestra final del estudio, con 55 madres y 13 padres, los cuales tenían a su cargo un total de 134 hijos 
entre todos. Las características de los padres, de los hijos y de las familias se recogen en la tabla 1 .

\section{Instrumentos}

Cuestionario de Información Sociodemográfica. A través de este cuestionario se recogió información acerca del sexo del progenitor, edad, estado civil, nivel educativo, profesión, profesión del otro progenitor, número de hijos, sexo y edad de los mismos, alteraciones en el desarrollo, nivel de ingresos anuales por familia, convivencia de ambos educadores en el mismo hogar, horas semanales de ayuda doméstica y de ayuda en la crianza.

Escala abreviada de Depresión, Ansiedad y Estrés (DASS-21; Antony, Bieling, Cox, Enns, y Swinson, 1998). Se trata de un auto-informe breve de 21 ítems distribuidos en tres sub-escalas de 7 ítems cada una que miden la severidad de los síntomas de depresión, ansiedad y estrés. Los ítems son valorados de 0 (no se aplica nada a mí) a 3 (se aplica mucho a mí la mayor parte del tiempo). En cuanto a las propiedades psicométricas la escala original mostró adecuada consistencia interna $(\alpha=.87-.94)$, validez convergente con medidas de ansiedad y depresión, y capacidad para discriminar entre muestras clínicas y comunitarias (Antony et al., 1998). Las propiedades psicométricas de la versión española también han resultado satisfactorias (Fonseca-Pedrero, Paino, Lemos-Giráldez, y Muñiz, 2010).

Cuestionario para la Evaluación de Adoptantes, Cuidadores, Tutores y Mediadores (CUIDA; Bermejo et al., 2006). Se trata de un auto-informe de 189 ítems que valora aspectos psicológicos y habilidades relacionados con la capacidad de proporcionar atención y cuidados. Incluye la valoración de 14 variables relacionadas con la personalidad de los cuidadores: Altruismo, Apertura, Asertividad, Autoestima, Capacidad para resolver problemas, Empatía, Equilibrio emocional, Independencia, Flexibilidad, Reflexibilidad, Sociabilidad, Tolerancia a la frustración, Capacidad de establecer vínculos afectivos y Capacidad de resolución del duelo. Asimismo, el instrumento permite obtener 4 puntuaciones de segundo orden: Cuidado afectivo, Cuidado responsable, Sensibilidad hacia los demás y Agresividad. El test CUIDA presenta valores adecuados de consistencia interna para las diferentes sub-escalas y validez convergente con medidas de personalidad.

Escala de Satisfacción Familiar por Adjetivos (ESFA; Barraca, López-Yarto, y Olea, 2000). Se compone de 27 ítems que incluyen adjetivos para valorar las respuestas afectivas, positivas o negativas del individuo con relación a las interacciones familiares. La escala presentó buenas propiedades psicométricas en cuanto a consistencia interna, fiabilidad test-retest y validez convergente con otras medidas de satisfacción familiar. 


\section{Procedimiento}

Para la evaluación de la eficacia del programa se empleó un diseño cuasiexperimental de un solo grupo con medidas pre-test y post-test. El muestreo utilizado fue de tipo no probabilístico incidental. Una vez realizada la selección de centros escolares, empresas, escuelas infantiles y centros de psicología, se contactó con los equipos directivos para ofertar la posibilidad de impartir un programa de entrenamiento a padres y la consiguiente participación en el estudio y a su vez se solicitó el consentimiento por escrito a los padres participantes antes de la impartición del programa. Todos los datos recogidos fueron estrictamente anónimos y se cumplió lo establecido en la Ley Orgánica 3/2018, de 5 de diciembre, de Protección de Datos Personales y garantía de los derechos digitales. Todos los procesos fueron revisados y autorizados por el comité de ética de la Universidad Católica de Murcia.

\section{Formato e intervención}

El programa de entrenamiento a padres Educar con una sonrisa es un modelo estructurado de intervención dirigido a padres de hijos en edades comprendidas entre 0 y 12 años, compuesto por 4 sesiones de intervención de dos horas, agrupadas en 3 módulos, impartido con una frecuencia semanal y administrado bajo un formato grupal de entre 5 y 10 personas. Esta intervención con grupos reducidos se debió a una estrategia elaborada con el fin de aumentar la eficacia del programa para la reducción de la sintomatología clínica. La persona que imparte el programa de entrenamiento debe ser un psicólogo experto en psicología clínica y educativa, con formación en el manejo de dinámicas grupales, ya que una de las funciones principales del terapeuta es actuar como "facilitador meta-cognitivo"competencia consistente en la modificación del pensamiento de aquellos participantes que muestren dificultades cognitivas y meta-cognitivas ante reflexiones sobre sus propios estados mentales (Inchausti, García-Poveda, Prado-Abril, Ortuño-Sierra, y Gaínza-Tejedor, 2017).

El programa sigue un modelo de intervención psicoeducativo compuesto por entrenamientos cognitivo-conductuales, instrucción en habilidades de crianza, dinámica de grupo, reflexiones conjuntas, ejercicios de relajación y movilización que se realizan en todas las sesiones. Para facilitar la implementación del programa, se estructuró el contenido en 17 estrategias, 13 principios de crianza efectiva y 34 características de autoridad, así mismo, se entregaron 5 documentos como material de seguimiento de las explicaciones. Con el fin de asentar los conocimientos adquiridos se entregó a los participantes unas lecturas y unas actividades extraídas del libro Educar con una sonrisa (Pérez-Saussol, 2015).

El programa de entrenamiento Educar con una sonrisa centra sus objetivos en: (1) aumentar la salud física y psicológica de los padres, (2) instruir en habilidades de crianza y cambio de actitud a los padres y (3) ayudar a los padres a mejorar la adaptación 
y funcionamiento psicosocial de sus hijos. En la tabla 3 se presenta la estructura completa del programa, los módulos con sus sesiones correspondientes, las estrategias, los principios de crianza efectiva y los documentos entregados.

\section{Tabla 3. Estructura completa del programa de entrenamiento Educar con una sonrisa}

\begin{tabular}{|c|c|c|}
\hline $\begin{array}{l}\text { Módulo } 1 . \\
\text { La Salud de los } \\
\text { padres. }\end{array}$ & $\begin{array}{c}\text { Sesión } 1 . \\
\text { Mejora del } \\
\text { estado físico y } \\
\text { psicológico de } \\
\text { los padres. }\end{array}$ & $\begin{array}{l}\text { Estrategias: Auto-recompensa (1): premiar el propio esfuerzo, Sonrisa (2): forzar la } \\
\text { sonrisa para provocar un cambio de actitud, Hacer equipo (3): utilizar las } \\
\text { competencias individuales a fin de conseguir una fuerza colectiva, } \\
\text { Autoconocimiento (4):conseguir una percepción real de nosotros mismos, } \\
\text { Normalización (5): eliminar el carácter de excepcionalidad de una situación, } \\
\text { Potenciación (6): reforzar y hacer visibles las propias capacidades, Rutinas } \\
\text { saludables (7): realizar actividades beneficiosas para la salud, Yo elijo (7): adquirir } \\
\text { la conciencia de agentes responsables del cambio, Resolución (8): intervenir en la } \\
\text { resolución de conflictos atendiendo a las parcelas de responsabilidad propia, } \\
\text { Relajación (9): tomar conciencia del cuerpo en distensión y de la mente en calma. } \\
\text { Documento: Cuestionario de salud para padres (1). }\end{array}$ \\
\hline \multirow[t]{2}{*}{$\begin{array}{l}\text { Módulo } 2 . \\
\text { Habilidades de } \\
\text { crianza y cambio } \\
\text { de actitud. }\end{array}$} & $\begin{array}{c}\text { Sesión } 2 . \\
\text { Entrenamiento } \\
\text { en habilidades } \\
\text { de crianza y } \\
\text { cambio de } \\
\text { actitudes I }\end{array}$ & $\begin{array}{l}\text { Estrategias: Aceptación (10): asimilar las circunstancias personales, } \\
\text { Empoderamiento (11): ensalzar las capacidades y habilidades propias, Movilización } \\
\text { (12): realizar movimientos corporales para activar la energía corporal. } \\
\text { Principios: Dedicar tiempo de calidad (1), Valorar y reconocer (2), Respetar el } \\
\text { desarrollo madurativo - no comparar (3), Facilitar que asuman responsabilidades } \\
\text { (4), Escuchar- prestar atención (5), Comunicar de forma clara y precisa (6). } \\
\text { Documentos: Claves de mejora para padres (2) y Principios de crianza (3). }\end{array}$ \\
\hline & $\begin{array}{c}\text { Sesión } 3 . \\
\text { Entrenamiento } \\
\text { en habilidades } \\
\text { de crianza y } \\
\text { cambio de } \\
\text { actitudes II. }\end{array}$ & $\begin{array}{l}\text { Estrategias: Acogida (13): vivenciar la sensación de afecto y cuidado. } \\
\text { Principios: Asumir las responsabilidades (7), Coherencia y asertividad (8), } \\
\text { Eliminar la queja - actuar en positivo (9), Amabilidad y afecto (10), Orientar y } \\
\text { planificar (11), Establecer límites adecuados (12). } \\
\text { Características de autoridad: sinceridad, disciplina, respeto, responsabilidad, } \\
\text { marcar pautas, saber apoyar, firmeza, seguridad, capacidad de decisión, } \\
\text { objetividad, respeto a la norma, justicia, coherencia, aceptación, serenidad y } \\
\text { calma, adecuación, humildad, tolerancia, valoración y reconocimiento, entrega } \\
\text { gratuita, capacidad de acogida, amabilidad, constancia, flexibilidad, capacidad de } \\
\text { escucha, sentido común, autocontrol, presencia- estar presente, compromiso, } \\
\text { equilibrio y estado emocional positivo. } \\
\text { Documentos: Principios de crianza (3) y Características de autoridad (4). }\end{array}$ \\
\hline $\begin{array}{l}\text { Módulo } 3 . \\
\text { Niveles de } \\
\text { adaptación y } \\
\text { funcionamiento } \\
\text { psicosocial del } \\
\text { niño. }\end{array}$ & $\begin{array}{c}\text { Sesión } 4 . \\
\text { Mejora del } \\
\text { funcionamiento } \\
\text { global del niño. }\end{array}$ & $\begin{array}{l}\text { Estrategias: Extrañeza (14): responder fingiendo sorpresa o asombro, Salir de la } \\
\text { escena (15): visualizar la situación conflictiva desde una perspectiva ajena, Nueva } \\
\text { oportunidad (16): responder ante una situación no adecuada permitiendo una nueva } \\
\text { versión de la acción anteriormente realizada, Apoyo (17): vivenciar la sensación de } \\
\text { protección y seguridad. } \\
\text { Principio: Proporcionar lo necesario (13). } \\
\text { Documento: Principios de crianza (3) y Necesidades de adaptación de los niños (5). }\end{array}$ \\
\hline
\end{tabular}

\section{Procedimiento y Contenido de las sesiones}

El procedimiento utilizado en la impartición de la sesiones fue muy parecido en todas ellas: (1) comienza la sesión (a partir de la segunda) con una revisión de las tareas asignadas para casa e invitando a la consulta de posibles dudas, (2) se continúa presentando los objetivos de la siguiente sesión, (3) se procede a realizar la psicoeducación correspondiente acompañada de los ejercicios, estrategias, principios y documentos que requiera, (4) se realiza una ronda de comentarios sobre los conceptos aprendidos, (5) se asignan unas tareas para casa.

Módulo 1: La salud de los padres. Está formado por la sesión 1 y su objetivo principal consiste en ayudar a los padres a reconocer su situación actual de salud física y 
psicológica; y en aportarles los recursos necesarios para incorporar rutinas saludables además de mejorar su bienestar psicológico. Cómo objetivos específicos se determinaron: (1) Presentar al terapeuta y el programa de entrenamiento. (2) Reflexionar sobre el rol de ser padres. (3) Exponer la importancia de cubrir las necesidades básicas de las figuras parentales. (4) Incorporar rutinas saludables en la vida de los padres. (5) Entrenar en las habilidades y estrategias que aparecen en la tabla 3. Los ejercicios que se llevaron a cabo fueron: (1) Presentación, (2) Relajación, (3) Rellenar un cuestionario de salud para padres.

Módulo 2: Habilidades de crianza y cambio de actitud. Está formado por la sesión 2 y 3 . El objetivo principal del módulo 2 consiste en dar a conocer los primeros 12 principios de crianza efectiva y la estrategia Acogida cuyos contenidos se recogen en la tabla 2. Como objetivos específicos se determinaron: (1) Instruir en habilidades parentales relacionadas con la dedicación de tiempo de calidad a los hijos. (2) Dar a conocer herramientas que facilitan la valoración y el reconocimiento y aumentan la seguridad de los niños. (3) Aportar herramientas para una comunicación adecuada. (4) Reflexionar sobre la responsabilidad parental. (5) Reflexionar sobre la fuerza de la coherencia y asertividad. (6) Ofrecer herramientas que faciliten eliminar la queja y actuar en positivo. (7) Insistir en los beneficios de mejorar la comunicación con los hijos. (8) Conocer y entrenar en las características de autoridad. (9) Evidenciar la importancia de imponer límites adecuados. Los ejercicios realizados son: (1) Relajación dirigida, (2) Movilización, (3) Rellenar un cuestionario de claves de mejora para padres, (4) Comenzar a cumplimentar un cuestionario de principios de crianza efectiva, (5) Rellenar un cuestionario sobre características de autoridad.

Módulo 3: Niveles de adaptación y funcionamiento psicosocial del niño. Está formado por la sesión 4 y su objetivo principal consiste en conocer el nivel actual de adaptación y el funcionamiento psicosocial de los hijos y enseñar a los padres a intervenir en su mejora. Como objetivos específicos se determinaron: (1) Instruir en estrategias para marcar los límites con seguridad y adecuación. (2) Dar herramientas para evaluar a sus hijos a nivel físico, afectivo-relacional e intelectual y enseñar estrategias de intervención. 3) Reflexionar sobre el de funcionamiento global de sus hijos. (4) Enseñar a los padres a realizar modificaciones cognitivas y conductuales. (5) Cerrar y despedir el programa de entrenamiento. Todos estos contenidos se encuentran recogidos en la tabla 3. Los ejercicios realizados son: (1) Apoyo, (2) Despedida y cierre.

\section{Análisis de datos}

Para realizar el análisis de datos se calcularon estadísticos descriptivos para las variables demográficas y de resultado. El análisis de normalidad de las variables 
cuantitativas se llevó a cabo a través del análisis exploratorio y el Test Kolmogorov-Smirnov. Debido a que los resultados mostraron diferencias significativas $(p=.00)$ entre la distribución de las variables objeto de estudio y la distribución normal, se utilizó el Test de Wilcoxon para la comprobación de las diferencias entre pre-test y post-test. Finalmente para la comprobación del efecto de los niveles iniciales de severidad en la eficacia del programa sobre las variables clínicas, se llevaron a cabo modelos de regresión lineales donde se calculó el coeficiente de regresión $\beta_{1}$, el estadístico de significación $t$ de Student y el coeficiente de determinación $R^{2}$ para cada una de las variables.

\section{RESULTADOS}

De los 98 padres que iniciaron el programa de entrenamiento, 68 fueron los que terminaron formando la muestra definitiva del estudio, por lo que la adherencia al programa fue del $69.4 \%$. En la tabla 4 se muestran los estadísticos descriptivos del pretest, como se puede observar, las medidas en las variables clínicas se encontraban en el rango subclínico, mientras que las habilidades en relación con la crianza y la satisfacción familiar presentaron puntuaciones dentro del rango de la población general.

Eficacia para la mejora de síntomas clínicos, satisfacción familiar y habilidades en relación con la crianza

Los síntomas clínicos relacionados con la ansiedad, la depresión y el estrés mostraron mejoras significativas, como también ocurrió con la satisfacción familiar, la cual vio su puntuación incrementada significativamente tras la implementación del programa y las siguientes habilidades en relación a la crianza: apertura, asertividad, autoestima, capacidad de resolver problemas, empatía, equilibrio emocional, independencia, flexibilidad, reflexividad, sociabilidad, tolerancia a la frustración, capacidad de establecer vínculos afectivos, capacidad de resolución del duelo, cuidado responsable, cuidado afectivo y sensibilidad hacia los demás, observándose a su vez una reducción significativa en la agresividad. Datos que se pueden contrastar en la tabla 4.

Severidad de los síntomas clínicos como moderador de la eficacia del programa

Debido a que el programa de entrenamiento fue aplicado en una muestra de padres sin patología diagnosticada, la severidad promedio en ansiedad, depresión y estrés antes de asistir al programa de entrenamiento se situaba en un rango subclínico. A partir de estos resultados, se procedió a comprobar si los niveles iniciales alcanzados en estas variables ejercían efectos significativos sobre las variaciones que experimentaban dichas variables debido a la eficacia del programa. Con el fin de comprobar la influencia 
de los niveles iniciales de las variables clínicas sobre la puntuación de cambio tras la recepción del programa (diferencia entre la puntuación pre-test y la puntuación posttest), se estimaron modelos de regresión lineal simple para cada una de las variables, utilizando como predictor la puntuación de cada variable en el pre-test y su puntuación de cambio como variable dependiente. La ecuación de regresión a estimar para cada variable fue la siguiente: $\Delta \mathrm{X}=\beta_{0}+\beta_{1} \mathrm{X}^{\mathrm{PRE}}+\boldsymbol{\varepsilon}$

Tabla 4. Medias, desviaciones típicas y resultados del test de Wilcoxon

\begin{tabular}{|c|c|c|c|c|c|c|}
\hline Variables & & $\begin{array}{c}\text { Media }(D T) \\
\text { Pre-test }\end{array}$ & $\begin{array}{c}\text { Media }(D T) \\
\text { Post-test }\end{array}$ & $\begin{array}{c}\text { Media }(D T) \\
\text { Cambio }\end{array}$ & $Z$ & $p$ \\
\hline \multirow{3}{*}{ Clínicas } & Depresión & $3.18(3.60)$ & $1.32(1.66)$ & $-1.85(2.69)$ & -5.26 & .00 \\
\hline & Ansiedad & $2.37(2.83)$ & $1.04(1.53)$ & $-1.32(2.46)$ & -4.02 & .00 \\
\hline & Estrés & $7.10(4.59)$ & $4.38(3.59)$ & $-2.72(4.11)$ & -5.03 & .00 \\
\hline Satisfacción & Satisfacción familiar & $125.54(20.01)$ & $138.37(17.81)$ & $12.82(12.93)$ & 6.57 & .00 \\
\hline \multirow{18}{*}{$\begin{array}{l}\text { Habilidades } \\
\text { de crianza }\end{array}$} & Altruismo & $4.19(1.50)$ & $4.21(1.91)$ & $.15(1.42)$ & 0.07 & .94 \\
\hline & Apertura & $4.62(2.10)$ & $5.35(2.04)$ & $.73(1.42)$ & 3.76 & .00 \\
\hline & Asertividad & $3.91(2.06)$ & $4.88(1.86)$ & $.97(1.40)$ & 4.81 & .00 \\
\hline & Autoestima & $3.90(1.98)$ & $5.28(1.67)$ & $1.38(1.29)$ & 6.27 & .00 \\
\hline & Capacidad de resolver problemas & $3.29(2.09)$ & $4.22(2.04)$ & $.93(1.24)$ & 5.24 & .00 \\
\hline & Empatía & $4.31(1.96)$ & $5.00(1.84)$ & $.69(1.45)$ & 3.59 & .00 \\
\hline & Equilibrio emocional & $3.12(1.79)$ & $4.29(2.03)$ & $1.18(1.17)$ & 5.83 & .00 \\
\hline & Independencia & $4.19(1.91)$ & $4.63(2.09)$ & $.44(1.56)$ & 2.25 & .02 \\
\hline & Flexibilidad & $3.44(2.04)$ & $3.96(2.12$ & $.51(1.77)$ & 2.30 & .02 \\
\hline & Reflexividad & $3.35(1.81)$ & $3.82(1.66)$ & $.47(1.35)$ & 2.73 & .02 \\
\hline & Sociabilidad & $4.24(2.03)$ & $4.87(1.87)$ & $.63(1.58)$ & 3.52 & .00 \\
\hline & Tolerancia a la frustración & $3.35(1.73)$ & $4.41(1.93)$ & $1.06(1.48)$ & 4.99 & .00 \\
\hline & Capacidad de establecer vínculos afectivos & $3.96(1.93)$ & $4.60(1.90)$ & $.65(1.35)$ & 3.65 & .00 \\
\hline & Capacidad resolución del duelo & $4.54(1.21)$ & $5.18(2.13)$ & $.63(1.36)$ & 3.48 & .00 \\
\hline & Cuidado responsable & $2.90(1.95)$ & $3.90(1.98)$ & $.95(1.25)$ & 5.33 & .00 \\
\hline & Cuidado afectivo & $3.69(2.06)$ & $4.93(2.08)$ & $1.23(1.35)$ & 5.65 & .00 \\
\hline & Sensibilidad hacia los demás & $3.51(1.86)$ & $4.40(1.88)$ & $.88(1.19)$ & 5.22 & .00 \\
\hline & Agresividad & $6.94(1.91)$ & $5.21(1.94)$ & $-1.73(1.60)$ & -6.10 & .00 \\
\hline
\end{tabular}

Donde $\Delta \mathrm{X}$ fue la diferencia entre las puntuaciones postest y pretest, $\mathrm{X}^{\mathrm{PRE}}$ las puntuaciones iniciales de la variable clínica, $\beta_{0}$ fue utilizado como el término constante de la recta de regresión, $B_{0}$ la pendiente de la recta de regresión y, por lo tanto, reflejó como variaba $\Delta \mathrm{X}$ cuando cambiaba en una unidad la variable $\mathrm{X}^{\mathrm{PRE}} ; \varepsilon$ fue utilizado para el término de error, el cual recogió las diferencias entre los datos y la predicción del modelo de regresión. Para la comprobación de la significación estadística del coeficiente de regresión se llevaron a cabo pruebas $t$ student bajo la hipótesis nula de $\beta_{l}=0$. Por último, se obtuvo el coeficiente de determinación $R^{2}$ que indicó el porcentaje de varianza explicado de la puntuación de cambio por el nivel de la variable en el pre-test.

Como se puede observar en la tabla 5, todos los coeficientes de regresión resultaron significativos, lo que indicó una asociación entre los niveles iniciales de severidad en cada una de las variables clínicas y el cambio experimentado tras el programa. El sentido negativo de los coeficientes indicó que a mayor severidad de los síntomas, las mejoras experimentadas fueron mayores, con un porcentaje de varianza 
explicada del $78 \%$ para los síntomas depresivos, del $67 \%$ para la ansiedad y del $44 \%$ para el estrés.

Tabla 5. Estimación de los coeficientes de regresión para la severidad inicial en las variables clínicas en función de la puntuación de cambio

\begin{tabular}{lcccc}
\hline Variables clínicas & $\beta_{1}$ & $T$ & $p$ & $R^{2}$ \\
\hline Depresión & -.65 & -13.86 & .00 & .78 \\
\hline Ansiedad & -.7375 & -10.07 & .00 & .67 \\
\hline Estrés & -.6117 & -7.072 & .00 & .44 \\
\hline
\end{tabular}

\section{DISCUSIÓN Y CONCLUSIONES}

El presente estudio aporta información acerca de la viabilidad y eficacia del programa de entrenamiento para padres Educar con una sonrisa para la mejora del bienestar psicológico, la satisfacción familiar y las habilidades y dimensiones cognitivas relacionadas con la crianza en padres de niños entre 0 y 12 años.

Del total de padres de 98 padres que iniciaron el programa se obtuvo una tasa de adherencia del $69.4 \%$, quedando una muestra total de 68 padres, dato que resulta indicativo de los beneficios que reportan los programas breves de entrenamiento para el aumento de la adherencia a los programas y el seguimiento de los asistentes, facilitando la eliminación de impedimentos que presentan los programas de mayor duración, entre los que se encuentran: la modificación de horarios, la falta de tiempo y la atención a los hijos (Whittaker y Cowley, 2012).

Con relación a la eficacia del programa se han encontrado resultados estadísticamente significativos en las medidas de las variables clínicas. Estos hallazgos son similares a los observados en general para estudios acerca de programas de crianza que han utilizado medidas de resultado de tipo clínico (Bennett et al., 2013). Así mismo el efecto del programa sobre las variables Ansiedad, Depresión y Estrés se asoció significativamente a los niveles de severidad iniciales, observándose un mayor efecto cuando dichos niveles eran más elevados. En este sentido, los hallazgos de este estudio apoyan la idea del uso de programas de entrenamiento para la mejora del ajuste psicosocial parental, no solamente obtenida a través de la mejora de sus habilidades parentales, sino también, a través de la inclusión de componentes de intervención para abordar aspectos clínicos como puede ser el entrenamiento cognitivo, las estrategias de autorregulación emocional y la relajación como indicó Toro (2019) en sus trabajos.

Las medidas obtenidas en satisfacción familiar también se han visto incrementadas en las puntuaciones post-test. A su vez, tras examinar los efectos encontrados en las habilidades y dimensiones cognitivas en relación con la crianza obtenidas a través del test CUIDA (Bermejo et al., 2006), se observaron cambios significativos tanto en los factores de primer orden (Apertura, Asertividad, Autoestima, Capacidad de resolver problemas, Empatía, Equilibrio emocional, Independencia, 
Flexibilidad, Reflexividad, Sociabilidad, Tolerancia a la frustración, Capacidad de establecer vínculos afectivos y Capacidad resolución del duelo), como en las dimensiones de segundo orden (Cuidado responsable, Cuidado afectivo, Sensibilidad hacia los demás y Agresividad). La literatura científica sugiere que el aumento de dichas habilidades y dimensiones cognitivas provocan consecuencias en el bienestar psicológico de los padres (Pérez-Adame et al., 2013). A partir de los datos obtenidos en el presente estudio, podemos concluir que la mejora en estas habilidades y dimensiones cognitivas relacionadas con la crianza se han visto acompañadas de mejoras en la sintomatología clínica y la satisfacción familiar. Sin embargo, estos resultados no permiten establecer con certeza si los efectos en el ajuste psicosocial de los padres son el resultado de las mejoras experimentadas en la satisfacción familiar y las habilidades en relación a la crianza para el manejo de la conducta de los niños, o por el contrario, son consecuencia de las estrategias incluidas en el programa para el abordaje directo del bienestar psicológico parental.

Las limitaciones del presente estudio abren una línea a nuevas líneas de investigación. En primer lugar, el uso de un diseño cuasi-experimental con ausencia de grupo control indica una cautelosa interpretación de los resultados de eficacia ya que a pesar de ser prometedores, requerirían de un estudio experimental. En segundo lugar, el reducido tamaño de la muestra y su carácter incidental no permiten establecer conclusiones definitivas acerca de la generalización de los efectos del programa, quedando pendiente su evaluación en un estudio con un mayor número de participantes, seleccionados de forma aleatoria. Por último, sería recomendable realizar una evaluación previa y posterior a la realización del programa de entrenamiento de los padres que posibilitase la valoración de los efectos del programa en los niños y comprobar cómo la salud mental de los padres tiene influencia en el comportamiento de los niños (Maggi et al., 2010).

Las posibles aplicaciones del programa de entrenamiento Educar con una sonrisa en relación a los resultados obtenidos abren la posibilidad de utilizar este programa como una herramienta: (1) para la prevención ante posibles dificultades de tipo psicológico en los padres durante la etapa de crianza, (2) clínica para la mejora de la sintomatología clínica relacionada con la ansiedad, la depresión y el estrés, (3) para la mejora de la satisfacción familiar, (4) para la mejora de las habilidades y dimensiones cognitivas en relación con la crianza, (5) para dar formación tanto a profesores como a psicólogos que trabajen con padres o con niños y, por último, (6), este programa de entrenamiento a padres podría ser ofertado tanto a psicólogos como a profesores de centros educativos y otras instituciones relacionadas con la infancia. 


\section{REFERENCIAS}

Antony, M.M., Bieling, P.J., Cox, B.J., Enns, M.W., y Swinson, R.P. (1998). Psychometric properties of the 42-item and 21-item versions of the Depression Anxiety Stress Scales in clinical groups and a community sample. Psychological Assessment, 10(2), 176-181. doi: 10.1037/1040-3590.10.2.176

Armstrong, M.I., Birnie-Lefcovitch, S., y Ungar, M.T. (2005). Pathways between social support, family wellbeing, quality of parenting, and child resilience: What we know. Journal of Child and Family Studies, 14(2), 269-281. doi:10.1007/s10826-005-5054-4

Barlow, J., Smailagic, N., Ferriter, M., Bennett, C., y Jones, H. (2016). Group-based parent-training programmes for improving emotional and behavioural adjustment in children from birth to three years old. Cochrane Database of Systematic Reviews, 8, 1-94. doi:10.1002/14651858.CD003680.pub2

Barraca, J., y López-Yarto, L. (1999). ESFA: Escala de satisfacción familiar por adjetivos. TEA Ediciones.

Barraca, J., Yarto, L.L., y Olea, J. (2000). Psychometric properties of a new family life satisfaction scale. European Journal of Psychological Assessment, 16(2), 98-106. doi:10.1027//1015-5759.16.2.98

Bennett, C., Barlow, J., Huband, N., Smailagic, N., y Roloff, V. (2013). Group-based parenting programs for improving parenting and psychosocial functioning: A systematic review. Journal of the Society for Social Work and Research, 4(4), 300-332. doi: $10.5243 /$ jsswr.2013.20

Bermejo, F.A., Estévez, I., García, M.I., García-Rubio, E., Lapastora, M., Letamendía, P., ... y de Castro, F.V. (2006). Cuestionario para la evaluación de adoptantes, cuidadores, tutores y mediadores. TEA Ediciones.

Bloomfield, L., y Kendall, S. (2012). Parenting self-efficacy, parenting stress and child behavior before and after a parenting program. Primary Health Care Research $y$ Development, 13(4), 364-372. doi:10.1017/S1463423612000060

Centro de investigaciones sociológicas - CIS- (2012). Familia y género. Recuperado de: http://www.cis.es/cis/export/sites/default/Archivos/Marginales/2940_2959/2942/es2942. pdf.

Chambless, D.L., y Hollon, S. (1998). Defining empirically-supported therapies. Journal of Consulting and Clinical Psychology, 66, 7-18. doi:10.1037//0022-006x.66.1.7

Crandall, A., Deater-Deckard, K., y Riley, A.W. (2015). Maternal emotion and cognitive control capacities and parenting: A conceptual framework. Developmental Review, 36, 105-126. doi: 10.1016/j.dr.2015.01.004

Eamesa, C., Daley, D., Hutchings, J., Whitaker, C.J., Bywater, T., Jones, K., y Hughes, J.C. (2010). The impact of group leaders' behaviours on parent's adquisition of key parenting skills during parent training. Behaviour Research and Therapy, 48, 1221-1226. doi:10.1016/j.brat.2010.07.011

Flores-Bravo, J.F., Valadez-Sierra, M.D., Rosal, A.B., y Betancourt-Morejón, J. (2018). Principales preocupaciones de padres de hijos con altas capacidades. Revista de Educación y Desarrollo, 47, 115-122.

Fonseca-Pedrero, E., Paino, M., Lemos-Giráldez, S. y Muñiz, J. (2010). Propiedades psicométricas de la Depression, Anxiety and Stress Scales-21 (DASS-21) en universitarios españoles. Ansiedad y-Estrés, 16(2), 215-226.

Furlong, M., McGilloway, S., Bywater, T., Hutchings, J., Smith, S.M., y Donnelly, M. (2012). Behavioural and cognitive-behavioural group-based parenting programmes for 
early-onset conduct problems in children aged 3 to 12 years. Campbell Systematic Reviews, 8(1), 1-239. doi:10.1002/14651858.CD008225.pub2

Godoy, J. F., Ruiz Castilla, M., Fresneda López, M.D., y Puertas González, J.A. (2019). Eficacia de un programa de entrenamiento para padres destinado a mejorar la comunicación oral y la conducta de sus hijos: un estudio preliminar. Revista de Investigación en Logopedia, 9(2), 107-127. doi: 10.5209/rlog.62544

González, J.A.P., Castilla, M.R., López, M.D.F., y Godoy, J.F. (2019). Eficacia de un programa de entrenamiento para padres destinado a mejorar la comunicación oral y la conducta de sus hijos: un estudio preliminar. Revista de Investigación en Logopedia, 9(2), 107-127.

Hermanns, J.M., Assecher, J.J., Zijlstra, B.J., Hoffenaar, P.J., y Dekovic, M. (2013). Long-term changes in parenting and child behavior after the Home-Start family support program. Children and Youth Services Review, 35, 678-684. doi: 10.1016/j.childyouth.2013.01.017

Hiscock, H., Bayer, J. K., Price, A., Ukoumunne, O. C., Rogers, S., y Wake, M. (2008). Universal parenting programme to prevent early childhood behavioural problems: cluster randomised trial. British Medical Journal, 336, 318-321. doi: 10.1136/bmj.39451.609676.AE

Inchausti, F., García-Poveda, N.V., Prado-Abril, J., Ortuño-Sierra, J., y Gaínza-Tejedor, I. (2017). Entrenamiento en habilidades sociales orientado a la metacognición (MOSST): Marco teórico, metodología de trabajo y descripción del tratamiento para pacientes con esquizofrenia. Papeles del Psicólogo, 38(3), 204-215.

Jones, J.D., Lebowitz, E.R., Marin, C.E., y Stark, K.D. (2015). Family accommodation mediates the association between anxiety symptoms in mothers and children. Journal of Child $y$ Adolescent Mental Health, 27(1), 41-51. doi:10.2989/17280583.2015.1007866

Kjøbli, J., Hukkelberg, S.Y., y Ogden, T. (2013). A randomized trial of group parent training: Reducing child conduct problems in real-world settings. Behaviour, 2, 1-10.

Kjøbli, J., y Ogden, T. (2009). Gender differences in intake characteristics and behavior change among children in families receiving parent management training. Children and Youth Services Review, 31, 823-830. doi:10.1016/j.childyouth.2009.03.004

Leijdesdorff, S., van Doesum, K., Popma, A., Klaassen, R., y van Amelsvoort, T. (2017). Prevalence of psychopathology in children of parents with mental illness and/or addiction: an up to date narrative review. Current Opinion in Psychiatry, 30(4), 312-317. doi: 10.1097/YCO.0000000000000341

López de Arana Prado, E., y Barandiaran Arteaga, A. (2018). Vivencias sobre la maternidad: aspectos que tener en cuenta en la promoción de la salud infantil. Pediatría Atención Primaria, 20(79), 237-243.

Lozano-Rodríguez, I., y Valero-Aguayo, L. (2017). Una revisión sistemática de la eficacia de los programas de entrenamiento a padres. Revista de Psicología Clínica con Niños y Adolescentes, 4(2), 85-91. doi:10.17060/ijodaep.2014.n1.v4.607

Maggi, S., Irwin, L.J., Siddiqi, A., y Hertzman, C. (2010). The social determinants of early child development: an overview. Journal of Pediatrics and Child Health, 46(11), 627-635. doi: 10.1111/j.1440-1754.2010.01817.x

Manuel, J., Casanova, P.F., Carpio, M.V., y Cerezo, M.T. (2015). Consistencia e inconsistencia parental: relaciones con la conducta agresiva y satisfacción vital de los adolescentes. European Journal of Education and Psychology, 6(2). doi:10.1989/ejep.v6i2.112

Martin, A.J., y Sanders, M.R. (2003). Balancing work and family: A controlled evaluation of the Triple P-Positive Parenting Program as a work-site intervention. Child and Adolescent Mental Health, 8(4), 161-169. doi:10.1111/1475-3588.00066 
Matsumoto, Y., Sofronoff, K., y Sanders, M.R. (2010). Investigation of the effectiveness and social validity of the Triple P Positive Parenting Program in Japanese society. Journal of Family Psychology, 24(1), 87-91. doi:10.1037/a0018181

McMahon, R.J. (1991). Entrenamiento de padres.V. Caballo, Manual de Técnicas de Terapia y Modificación de Conducta. Madrid: Siglo XXI.

Mendoza, B., Pedroza, F.J., y Martínez, K.I. (2014). Prácticas de Crianza Positiva: Entrenamiento a padres para reducir Bullying. Acta de Investigación Psicológica, 4(3), 1793-1808. doi: 10.1016/S2007-4719(14)70980-9

Middeldorp, C.M., Wesseldijk, L.W., Hudziak, J.J., Verhulst, F.C., Lindauer, R.J., y Dieleman, G.C. (2016). Parents of children with psychopathology: psychiatric problems and the association with their child's problems. European Child \& Adolescent Psychiatry, 25(8), 919-927. doi:10.1007/s00787-015-0813-2

Otani, K., Suzuki, A., Matsumoto, Y., Shibuya, N., Sadahiro, R., y Enokido, M. (2013). Parental overprotection engenders dysfunctional attitudes about achievement and dependency in a gender specific manner. BMC Psychiatry, 13, 1-4. doi:10.1186/1471-244X-13-345

Padilla, J.P., Lara, B.L., y Álvarez-Dardet, S.M. (2010). Estrés y competencia parental: un estudio con madres y padres trabajadores. Suma Psicológica, 17(1), 47-57.

Patterson, G. (1982). Coercive family process. Eugene, OR: Castalia.

Patterson, G., Reid, J., Jones, R., y Conger, R. (1975). A social learning approach to family intervention. Vol. 1: Families with aggressive children. Eugene, OR: Castalia.

Pérez, F., y Santelices, M.P. (2016). Sintomatología depresiva, estrés parental y funcionamiento familiar. Revista Argentina de Clínica Psicológica, 25(3), 235-244.

Pérez-Adame, E., Fulgencio-Juárez, M., y González-Zepeda, A.P. (2013). Burnout en personal de estancias infantiles y su relación con las habilidades y conocimientos requeridos para el puesto. Revista de Educación y Desarrollo, 24, 5-12.

Perez-Saussol, C. (2015). Educar con una sonrisa. Murcia: Autoedición.

Porzig-Drummon, R., Stevenson, R.J., y Stevenson, C. (2014). The 1-2-3 Magic parenting program and its effect on child problem behaviors and dysfunctional parenting: A randomized controlled trial. Behaviour Research and Therapy, 58, 52-64. doi:10.1016/j.brat.2014.05.004

Porzig-Drummond, R., Stevenson, R.J., y Stevenson, C. (2015). Preliminary evaluation of a selfdirected video-based 1-2-3 Magic parenting program: A randomized controlled trial. Behaviour Research and Therapy, 66, 32-42. doi:10.1016/j.brat.2015.01.003

Raya, A.F., Pino, M.J., y Herruzo, J. (2009). La agresividad en la infancia: el estilo de crianza parental como factor relacionado. European Journal of Education and Psychology, 2(3), 211-222. doi:10.30552/ejep.v2i3.28

Rodrigo, M.J., Almeida, A., Spiel, C., y Koops, W. (2012). Introduction: Evidence-based parent education programmes to promote positive parenting. European Journal of Developmental Psychology, 9(1), 2-10. doi:10.1080/17405629.2011.631282

Sánchez, F. (2017). Conflictos entre padres e hijos: el problema de la disciplina. Revista de Formación Continuada de la Sociedad Española de Medicina de la Adolescencia, 1, 7080.

Sanders, M.R., Kirby, J.N., Tellegen, C.L., y Day, J.J. (2014). The Triple P-Positive Parenting Program: A systematic review and meta-analysis of a multi-level system of parenting support. Clinical Psychology Review, 34(4), 337-357. doi:10.1016/j.cpr.2014.04.003

Sanders, M.R., Turner, K.M., y Markie-Dadds, C. (2002). The development and dissemination of the Triple P-Positive Parenting Program: A multilevel, evidence-based system of parenting and family support. Prevention Science, 3(3), 173-189. doi:10.1023/a:1019942516231 
Shlonsky, A., Dennis, J.A., Devine, B., Tufford, L., Barlow, J., y Bjørndal, A. (2016). Mindfulness-based parenting programs for improving psychosocial outcomes in children from birth to age 18 and their parents. Cochrane Database of Systematic Reviews, 11, 1-21. doi:10.1002/14651858.CD012445

Toro, M. T. (2019). Relajación de jacobson para disminuir la ansiedad originada por evaluaciones e incremento del rendimiento académico en estudiantes de psicología de una universidad privada de Lima Metropolitana. Avances en Psicología, 27(2), 167-176.

Tosh, R., Arnott, W., y Scarinci, N. (2017). Parent-implemented home therapy programmes for speech and language: a systematic review. International journal of language \& communication disorders, 52(3), 253-269.

Van Aar, J.V., Asscher, J.J., Zijlstra, B.J., Dekovic, M., y Hoffenaar, P.J. (2015). Changes in parenting and child behavior after the home-start family support program: A 10 year follow-up. Children and Youth Services Review, 53, 166-175. doi:10.1016/j.childyouth.2015.03.029

Webster-Stratton, C. (2001). The incredible years: Parents, teachers, and children training series. Residential treatment for children y youth, 18(3), 31-45. doi: 10.1300/J007v18n03_04

Whittaker, K. A. \& Cowley, S. (2012). An effective programme is not enough: A review of factors associated with poor attendance and engagement with parenting support programmes. Children y Society, 26(2), 138-149. doi:10.1111/j.1099-0860.2010.00333.x

Recibido: 19 de septiembre de 2020

Recepción Modificaciones: 24 de octubre de 2020

Aceptado: 28 de noviembre de 2020 